\title{
Avaliação da Usabilidade dos Assistentes Virtuais Google Assistant e Siri com Foco em Usuários Idosos
}

\author{
Tiago Carneiro Gorgulho Mendes Barros \\ Universidade Federal de Itajubá \\ tiagocgorgulho@gmail.com
}

\author{
Rodrigo Duarte Seabra \\ Universidade Federal de Itajubá \\ rodrigo@unifei.edu.br
}

\begin{abstract}
Technologies have advanced quickly over the years, and with that, it becomes increasingly diverse every day. One technology increasingly being explored is virtual assistants, which emerged to make it easier for users to interact with their smartphones. Along with this technological advancement, the number of elderly people has grown exponentially in recent years, and tend to increase even more. Furthermore, this part of the population is having a greater interest in using these new technologies due to the ease generated. For this reason, they must be accessible to users of all ages. This research aims to evaluate the usability of Google Assistant and Siri virtual assistants, focusing on elderly users. The main results were that the elderly, even taking longer than the participants of the other groups evaluated, found that the virtual assistants facilitated the accomplishment of the tasks. Some factors that influenced the results of the performance of the elderly were the loss of cognitive and motor skills due to age and previous experience with the use of technology.
\end{abstract}

\section{KEYWORDS}

Usability, Older users, Virtual assistants, Siri, Google Assistant

\section{INTRODUÇÃO}

De acordo com uma pesquisa realizada pela Organização Mundial de Saúde, agência subordinada à Organização das Nações Unidas [1], a população mundial idosa, isto é, com mais de sessenta anos, será de dois bilhões até 2050, o que representará um quinto da população mundial. Isso mostra como o crescimento dessa parcela da população será acelerado, já que, atualmente, a população idosa é de, aproximadamente, 841 milhões de pessoas. Esse crescimento, portanto, será de cerca de $138 \%$ da população atual, em um espaço de tempo um pouco maior que três décadas. Segundo dados do Instituto Brasileiro de Geografia e Estatística [2], a parcela da população idosa brasileira que possui um telefone móvel é de $63,5 \%$, sendo que destes, $31,1 \%$ utilizam a Internet. Concatenando essas informações com o fato de que a população idosa brasileira era a quinta maior população idosa do mundo em 2016 [3], pode-se perceber que o nicho de mercado relativo à tecnologia, envolvendo pessoas acima de sessenta anos, crescerá nas próximas décadas.

Nielsen [4] defende que no início da Internet, este recurso era acessado apenas por pessoas que possuíam um conhecimento mais avançado da tecnologia. Todavia, com o crescimento da popularização da web, os usuários foram ficando cada vez mais diversificados, evidenciando-se a necessidade de se pensar em diretrizes de acessibilidade para esses usuários. Para Godinho [5], "acessibilidade consiste na facilidade de acesso e de uso de ambientes, produtos e serviços por qualquer pessoa e em diferentes contextos". Entretanto, um dos grupos que é amplamente afetado com a dificuldade de utilizar a tecnologia é o de idosos. Isso se deve ao fato de que, conforme Krug [6], indivíduos com 60 anos ou mais têm maior risco de declínio nas funções cognitivas por conta da maior exposição a fatores de risco [7][8] e do maior comprometimento nos mecanismos de percepção, raciocínio, memória [8][9], práxia, linguagem, atenção [10] e aprendizagem [11].

Para que haja maior inclusão do grupo de idosos na utilização das tecnologias em geral, deve-se buscar uma melhor usabilidade dos artefatos tecnológicos por meio de uma série de requisitos que devem ser seguidos. Conforme um relatório da Cisco [12], muitas vezes, as pessoas idosas não utilizam a tecnologia devido ao seu design. Para Betiol [13], a usabilidade pode ser definida como o grau de facilidade com que o usuário interage com uma interface específica. Para que se possa avaliar a facilidade de uso de uma interface, podem-se usar alguns componentes, como sugere Nielsen [14].

Para a realização do estudo em tela, que possui como públicoalvo idosos, foram utilizados os assistentes virtuais Google Assistant e Siri, presentes nos sistemas operacionais Android (7.0) e iOS (12.3.1), respectivamente. Esses assistentes virtuais, com base em dados coletados dos usuários, os auxiliam a realizar tarefas levando em consideração o contexto em que eles estão inseridos. Considerando que os assistentes virtuais podem ser utilizados via voz, eles são um recurso interessante aos usuários idosos, uma vez que tendem a diminuir as limitações causadas pelo envelhecimento [15].

Com base no exposto, o objetivo desse trabalho é avaliar a usabilidade dos assistentes virtuais Google Assistant e Siri, com foco em usuários idosos. Além deste grupo, foram utilizados outros dois grupos etários de usuários, visando comparar não só os dados obtidos pela comparação dos dois assistentes virtuais, mas também, entre os três grupos avaliados. Os grupos foram divididos da seguinte maneira, com base no estudo de de Lara [16]: jovens adultos (18-39 anos), adultos (40-59 anos) e idosos (idade igual ou superior a 60 anos). Para alcançar este objetivo, variadas tarefas foram propostas para os usuários realizarem, 
podendo-se chegar a conclusões sobre a usabilidade de ambos os assistentes virtuais com base nos diferentes grupos avaliados.

\section{FUNDAMENTAÇÃO TEÓRICA}

De acordo com Nielsen e Loranger [17], a usabilidade pode ser considerada como um atributo de qualidade referente à facilidade de utilizar algo. Entretanto, um dos problemas do termo "usabilidade" é que o mesmo pode ter uma variação de significados, dependendo de quem o utiliza. Até mesmo Nielsen [18] define a usabilidade de duas formas divergentes, sendo uma centrada nos fatores humanos, na ergonomia e na psicologia, e, a outra, em termos de funcionalidade, eficiência e confiabilidade. Ainda pelas definições de Nielsen [19], o autor propõe um conjunto de dez heurísticas. Jordan [20] apresenta a definição do termo "usabilidade" pautada na facilidade da utilização de determinado produto, porém, sem desconsiderar a classificação do termo proposto pela ISO 9241-11 (International Organization for Standardization), que definiu a usabilidade como a "medida na qual um produto pode ser usado por usuários específicos para alcançar objetivos específicos com eficácia, eficiência e satisfação em um contexto específico de uso" [21].

Whiteside, Bennett e Holtzblatt [22] ressaltam que a usabilidade serve como um conjunto de técnicas que auxiliam no desenvolvimento de interfaces de usuários. Já Bevan [23], como sendo um termo utilizado para descrever a qualidade da interação de um usuário com uma interface específica. Preece [24] defende que a usabilidade de um sistema se divide em possuir uma boa eficácia e eficiência em sua utilização, ser seguro no uso, ter uma boa utilidade para o usuário e ser de fácil aprendizagem e recordação. Já o autor Ben Shneiderman define oito "regras de ouro" [25], a saber: fornecer atalhos, perseguir a consistência, marcar o final dos diálogos, fornecer feedback informativo, permitir o cancelamento de ações, fornecer prevenção e manipulação simples de erros, reduzir a carga de memória de trabalho e fornecer controle e iniciativa do usuário.

$\mathrm{Na}$ atualidade, tendo em vista a diversidade de dispositivos móveis e o modo pelo qual os conteúdos são consumidos pelos usuários, há grandes desafios em relação às metodologias de projeto e avaliação da usabilidade [26]. É preciso, também, levar em consideração que os dispositivos móveis, em especial os smartphones, apresentam algumas limitações, por exemplo, o tamanho da tela, rede de Internet limitada em alguns casos, assim como os recursos de entrada, sendo preciso um pouco de habilidade motora, dentre outros. Essas limitações influenciam profundamente na maneira que os usuários idosos utilizam os smartphones e os recursos tecnológicos de modo geral.

\subsection{Idosos e Tecnologia}

Para uma pessoa ser considerada idosa, pela classificação do IBGE, é preciso que ela tenha mais de 65 anos, entretanto, para a realização desse estudo, será utilizada a classificação adotada pelo Estatuto do Idoso do Brasil, que considera uma pessoa idosa aquela que possui mais de 60 anos de idade (Lei № 10.741, de 01/10/2003).

As pessoas da terceira idade estão vivendo cada vez mais, sendo que esta população vem aumentando gradualmente ao longo dos anos. Essa mudança demográfica tem motivado pesquisadores de diversas áreas a encontrar formas de melhorar a qualidade de vida dos idosos, suavizando os efeitos do envelhecimento por meio de medicações e tecnologias assistivas [27]. Um estudo de Mamolo e Scherbov [28], focado na projeção da população nos países europeus, relata que haverá uma expansão da população idosa, enquanto o restante da população tenderá a diminuir. Ainda nessa projeção, é previsto que até o ano de 2030, uma pessoa entre quatro terá mais de 60 anos. Projeção semelhante foi realizada pelo IBGE [2] recentemente, prevendo que os idosos atingirão um quarto da população total no Brasil em 2060.

O crescimento da população idosa enfatiza a necessidade de se desenvolver abordagens inovadoras para auxiliá-los a utilizar novas tecnologias, transformando as pessoas desse grupo etário como potenciais usuários [29]. Os idosos são menos propensos a utilizarem dispositivos tecnológicos em relação aos mais jovens [30] e, por essa razão, não se pode esperar que conheçam algumas convenções adotadas para interfaces [31], além de interagirem de maneira diferente com a tecnologia por variadas razões. Sua cognição também é afetada, pois, no envelhecimento, há uma desaceleração dos processos cognitivos, na capacidade de memória, menor controle de atenção, diminuição da visão e audição, o que contribui para maior dificuldade no manuseio dos dispositivos móveis, além de resultar em erros cometidos ao interagir com a tecnologia [32].

Ao projetar tecnologias voltadas para idosos, é importante levar em consideração os sistemas perceptivos, cognitivos e motores desses usuários [33]. Além disso, quando a interação com a tecnologia é intensa ao longo da vida, a aprendizagem se torna mais fácil. Por outro lado, quando a tecnologia é introduzida mais tarde na vida de uma pessoa, seu uso e controle estarão menos associados às representações existentes que foram formadas durante a maturação neural [34].

\subsection{Assistentes Virtuais}

Os assistentes virtuais são agentes de interface autônomos que empregam métodos de raciocínio e inteligência adaptativos, fornecendo serviços ativos e colaborativos, além de assistências aos usuários em determinado aplicativo [35]. Os agentes de interface se diferem das interfaces usuais, pois é esperado que eles alterem seu comportamento e ações autonomamente mediante as ações do usuário e do estado que se encontra o sistema de acordo com a progressão da interação [36].

Os assistentes virtuais têm como principal objetivo proporcionar ao usuário melhor comunicação com um sistema, enfatizando a apresentação de certas informações e oferecendo recomendações personalizadas [37]. Para isso, eles utilizam um motor linguístico avançado que tenta, constantemente, identificar uma palavra chave, para que eles possam ser ativados. Uma vez que essa palavra chave é identificada, o assistente grava 
a voz do usuário e a envia para um servidor especializado que processará e interpretará o comando de voz. Esse servidor, para conseguir processar e interpretar a gravação do usuário, transformará a gravação em texto e a informação contida nele será processada. Dependendo do comando que o usuário requisitou, o servidor fornecerá ao assistente virtual as informações apropriadas para serem transmitidas de forma verbal e/ou textual para o usuário, reprodução de uma mídia solicitada, execução de tarefas, dentre outras ações [38]. O número de serviços que suportam os comandos de voz está crescendo cada vez mais rápido e os fabricantes de dispositivos de IOT (Internet of Things) também estão implementando, em seus produtos, essa tecnologia de controle de voz [39]. A tecnologia IOT permite a interconexão digital de objetos cotidianos com a Internet.

Embora cada assistente virtual possua alguns recursos exclusivos, eles compartilham algumas semelhanças entre si, sendo capazes de executar um conjunto de tarefas básicas, por exemplo: enviar e ler mensagens de texto (tanto via SMS como por aplicativos); enviar e ler e-mails; realizar e receber chamadas telefônicas; responder perguntas básicas como "Qual a previsão do tempo?" e "Que horas são?"; definir alarmes, lembretes e eventos no calendário; criar listas; controlar reprodução de vídeo em serviços conectados, tais como, Spotify, Netflix, YouTube; controlar dispositivos habilitados de IOT como alarmes, fechaduras e luzes; e contar piadas. Além de realizarem tarefas básicas como as anteriores, os assistentes virtuais podem adicionar outros recursos, conhecidos como "habilidades", que aumentam sua capacidade de customização por meio da interação com a interface de outros programas via comandos de voz [39]. Para a realização dessa pesquisa, os assistentes virtuais que serão utilizados são o assistente Siri e o Google Assistant, da Apple e do Google, respectivamente.

O Google Assistant é um assistente virtual desenvolvido pelo Google, projetado para auxiliar os usuários por meio da conversação. Ele é o sucessor do assistente Google Now, adicionando a função de se vincular com o Google Home, um alto-falante ativado por voz [40]. O Google Assistant se integra com diversas ferramentas que permitem ao usuário criar diversas habilidades para o assistente virtual. Um exemplo de habilidade que pode ser criada é que ao dizer "bom dia" ao Google Assistant, um conjunto de ações é iniciado visando otimizar a rotina do usuário, como ligar a cafeteira (desde que ela possua conexão com a Internet), ler as notícias, informar sobre a agenda do dia, informar a previsão do tempo etc. A Figura 1 ilustra a tela inicial do Google Assistant (à esquerda).

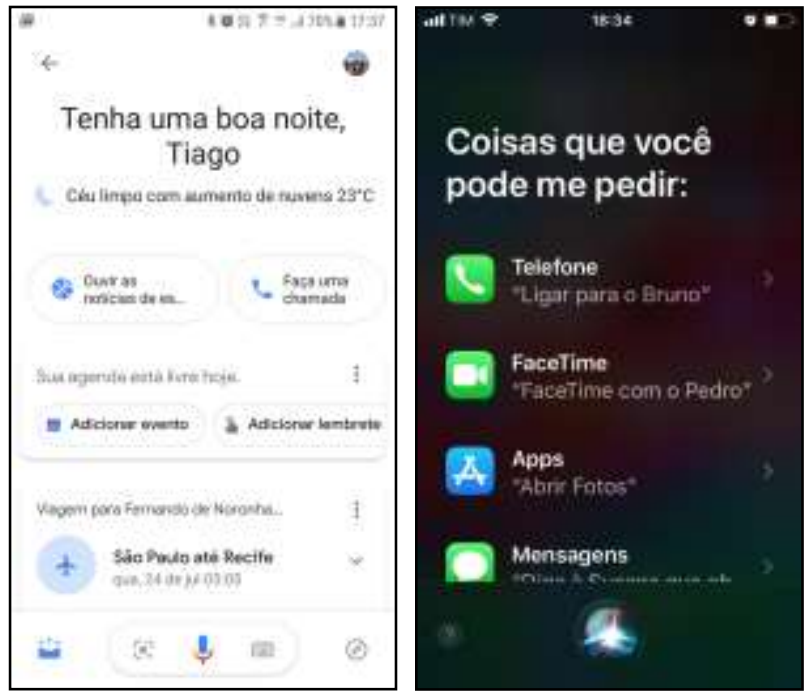

Figura 1: Tela inicial dos assistentes virtuais Google Assistant e Siri. Fonte: Os autores

A Siri é um assistente virtual lançado em 2010 para dispositivos da Apple, sendo o mais antigo dos assistentes virtuais. Atualmente, encontra-se em um processo de reformulação para a adição de novas funcionalidades e adaptação a novos dispositivos. A Siri pode antecipar algumas ações prevendo a necessidade do usuário, levando em consideração sua rotina. Ademais, por exemplo, pode indicar músicas ao usuário com base em seu gosto musical, localizar onde o carro foi estacionado, dentre muitas outras funcionalidades [41]. A Figura 1 (à direita) ilustra uma tela da Siri, informando algumas das possibilidades que o usuário pode solicitar.

De modo geral, quanto maior for a utilização dos assistentes virtuais, mais eles entenderão as necessidades e hábitos de seus usuários, conseguindo, ainda, reconhecer a voz do proprietário do dispositivo móvel, assim como identificar sotaques e dialetos diferentes. Esses recursos pode facilitar a interação de usuários da terceira idade, já que não é necessário usar a habilidade motora nem a visão para realizar determinadas tarefas.

\subsection{Trabalhos Correlatos}

Stößel et al. [42] realizaram uma pesquisa acerca da entrada de dados por meio dos gestos dos dedos, visando identificar se esse método de entrada é adequado para usuários idosos. O estudo comparou usuários idosos e jovens, analisando a velocidade e precisão de um conjunto de 42 gestos diferentes com os dedos em um dispositivo móvel. O nível de tamanho e a complexidade dos gestos variavam sistematicamente, visando verificar se interfeririam no desempenho dos usuários. Os resultados mostraram que os usuários idosos eram mais lentos, porém, não necessariamente menos precisos do que os usuários jovens. Os resultados observados indicam que esse método de entrada também é adequado para usuários idosos. 
Kurniawan [43] apresenta questões relacionadas ao uso de dispositivos móveis por usuários que possuem 60 anos ou mais de idade, e características que agradam estes usuários. Para realizar o estudo, foram utilizados métodos de análise quantitativa e qualitativa, discussões em grupo, entrevistas e uma pesquisa online. As entrevistas e as discussões dos grupos focais foram conduzidas para que se pudesse abranger os padrões de utilização, problemas, benefícios e recursos indesejados. As informações coletadas nessa etapa foram repassadas para uma pesquisa online realizada por um grupo de 100 pessoas. Chegou-se à conclusão que os usuários idosos são usuários passivos de dispositivos móveis, sentindo medo de usar tecnologias não familiares. Além disso, a maioria dos usuários preferiu recursos de design que auxiliam no declínio de suas habilidades funcionais.

Murata e Iwase [44] realizaram um trabalho sobre a usabilidade de interfaces touch com usuários idosos. Esse estudo utilizou três grupos de usuários: jovens, adultos de meia-idade e usuários idosos. Os resultados, levando em consideração o tempo de resposta dos usuários, mostraram que os usuários idosos apresentaram pior desempenho se comparados aos outros grupos quando foi utilizado um mouse no computador. Entretanto, quando foi utilizada uma tela touch, o tempo de resposta dos usuários idosos não teve diferença significativa aos dos outros grupos avaliados.

López et al. [45] conduziram um teste de usabilidade levando em consideração os assistentes virtuais, baseados em fala, mais prestigiados internacionalmente: Alexa, Cortana, Google Assistant e Siri. Uma comparação entre os serviços disponibilizados por cada assistente foi realizada com base nos seguintes aspectos: acesso a serviços de música, agenda, previsão do tempo, notícias, listas de tarefas e mapas. Os resultados mostraram que, embora exista uma gama de serviços disponíveis, há muito que se melhorar em relação à usabilidade desses sistemas.

Chiaradia, Seabra e Mattedi [46] realizaram um estudo sobre a usabilidade, com foco em usuários idosos, do assistente virtual Siri em dispositivos móveis. No estudo, três tarefas foram realizadas por participantes idosos e não idosos. Os autores concluíram que as pessoas idosas obtiveram um desempenho menor do que os outros participantes, face às suas condições motoras e cognitivas. Outro fator que influenciou o resultado do desempenho dos participantes foi o contato anterior com smartphones. Giassi e Seabra [47] analisaram a usabilidade da rede social Instagram com ênfase em usuários idosos usando smartphones. Foi proposta a realização de três tarefas em três grupos etários de participantes. Foi observado que os usuários idosos levaram cerca de cinco vezes mais tempo do que os outros usuários para executarem as mesmas tarefas.

A partir das pesquisas supramencionadas, nota-se que cada vez mais estudos nessa área estão sendo conduzidos, todavia, atualmente, ainda não há muitos trabalhos relativos à usabilidade de assistentes virtuais com enfoque em usuários idosos. Esse público tem crescido a cada dia em diversas partes do mundo e, por esse motivo, pesquisas envolvendo o uso de recursos tecnológicos por idosos se fazem necessárias.

\section{MÉTODO}

O trabalho foi realizado em duas cidades do Sul do Estado de Minas Gerais. Para a execução da pesquisa, foram propostas cinco tarefas distintas (T1, T2, T3, T4 e T5) aos participantes. Cada tarefa foi realizada em dois smartphones diferentes, um possuindo sistema operacional Android, para avaliar o assistente Google Assistant, e o outro com sistema operacional iOS, para avaliar a Siri. Nesse contexto, cada participante utilizou os dois smartphones. Este trabalho contou com a participação de 20 voluntários jovens adultos, de 18 a 39 anos (média $=26,1$ e desvio padrão $=4,21$ ), 20 voluntários adultos, de 40 a 59 anos (média = 48,9 e desvio padrão $=6,73$ ), e 20 voluntários idosos, com 60 anos ou mais de idade (média $=72,4$ e desvio padrão $=11,77$ ), totalizando 60 participantes.

Primeiramente, um questionário foi respondido pelos participantes com o objetivo de identificar seus perfis. No final do questionário havia um espaço para a assinatura dos participantes para registrar o consentimento do uso de seus dados, de maneira anônima, para a execução deste trabalho. Foi elucidado para os participantes que seriam mensurados os tempos para a execução de cada tarefa, sendo que a medição seria iniciada assim que o participante sinalizasse que poderia começar. A medição do tempo foi encerrada ao final de cada tarefa, assim que o participante afirmasse que o tempo poderia parar de ser contabilizado ou no momento que houvesse a desistência da tarefa. No final de cada uma das tarefas, um questionário, com perguntas fechadas, foi entregue a cada voluntário. Para esse questionário, foi utilizada uma escala Likert de cinco pontos nas respostas, visando identificar as opiniões relacionadas aos dois assistentes virtuais, bem como investigar a experiência vivenciada ao executar cada tarefa. Para não influenciar o desempenho dos participantes nas tarefas durante sua execução, o avaliador não respondeu qualquer pergunta. Essa conduta foi mantida mesmo no caso de perguntas relativas ao entendimento do que havia sido solicitado na tarefa.

\subsection{Critérios de Usabilidade}

Para Bosman e Charness [48], conhecer as capacidades e limitações dos usuários mais idosos auxilia na orientação dos designers e desenvolvedores para criar tecnologias mais acessíveis. À medida que as pessoas envelhecem, elas ganham algumas habilidades e perdem outras, entretanto, aprendem a compensar aquelas em declínio. Algumas dessas habilidades, como, por exemplo, a visão, influenciam diretamente na maneira como os usuários interagem com a tecnologia. Um exemplo no que tange às mudanças perceptivas das pessoas é apresentado por Fisk et al. [33], que defendem que aproximadamente metade da população masculina com mais de 65 anos e 30\% das mulheres sofrem com perda auditiva e, também, grande parte da população percebe problemas visuais por volta dos 40 anos de idade. 
Levando em consideração as limitações e as características dos idosos, bem como as pesquisas de Nielsen [14], Chiaradia, Seabra e Mattedi [46] e Giassi e Seabra [47], foram utilizadas as seguintes métricas para avaliar a usabilidade dos assistentes virtuais: tempo de aprendizagem, relativo ao tempo necessário para que o usuário aprenda a executar as atividades propostas e, também, de se familiarizar com a ferramenta em avaliação; desempenho, relacionado ao tempo que o usuário levará para executar as tarefas; taxa de erros cometidos pelo usuário, diz respeito aos erros cometidos, pelos usuários, durante a execução de cada tarefa, considerando a quantidade de erros e suas gravidades; sedimentação do conhecimento por experiência, relativo à facilidade para que um usuário possa reproduzir a mesma tarefa que foi realizada com base no que foi feito e aprendido anteriormente; satisfação subjetiva, métrica para analisar o nível de satisfação dos usuários ao utilizar a ferramenta analisada. No Quadro 1, pode-se encontrar os critérios de avaliação da usabilidade empregados nesta pesquisa.

\begin{tabular}{|c|c|c|}
\hline Critério & $\mathbf{N}^{\circ}$ & Perguntas \\
\hline \multirow{3}{*}{$\begin{array}{c}\text { Tempo de } \\
\text { Aprendizagem }\end{array}$} & 1 & Qual foi a facilidade de realizar a tarefa? \\
\hline & 2 & $\begin{array}{l}\text { As informações apresentadas na tela do } \\
\text { assistente virtual são facilmente identificadas e } \\
\text { compreendidas? }\end{array}$ \\
\hline & 3 & $\begin{array}{l}\text { As informações transmitidas verbalmente são } \\
\text { claras e fáceis de serem compreendidas? }\end{array}$ \\
\hline \multirow[b]{2}{*}{ Desempenho } & 4 & A tarefa foi simples de ser realizada? \\
\hline & 5 & $\begin{array}{l}\text { O assistente virtual tornou mais ágil a execução } \\
\text { da tarefa? }\end{array}$ \\
\hline \multirow{2}{*}{$\begin{array}{c}\text { Taxa de Erros } \\
\text { Cometidos pelo } \\
\text { Usuário }\end{array}$} & 6 & $\begin{array}{l}\text { Foi possível realizar a tarefa sem cometer } \\
\text { erros? }\end{array}$ \\
\hline & 7 & $\begin{array}{l}\text { Caso tenha cometido algum erro durante a } \\
\text { realização da tarefa, foi fácil revertê-lo? }\end{array}$ \\
\hline \multirow{2}{*}{$\begin{array}{l}\text { Sedimentação } \\
\text { do } \\
\text { Conhecimento } \\
\text { por Experiência }\end{array}$} & 8 & $\begin{array}{l}\text { O caminho percorrido para concluir a tarefa foi } \\
\text { intuitivo? }\end{array}$ \\
\hline & 9 & $\begin{array}{l}\text { Conseguiria realizar novamente a tarefa com a } \\
\text { ajuda do assistente virtual? }\end{array}$ \\
\hline \multirow{3}{*}{$\begin{array}{l}\text { Satisfação } \\
\text { Subjetiva }\end{array}$} & 10 & A interface do assistente virtual é atraente? \\
\hline & 11 & $\begin{array}{l}\text { A interface do assistente virtual possui muitas } \\
\text { informações desnecessárias para realizar a } \\
\text { tarefa? }\end{array}$ \\
\hline & 12 & Utilizaria novamente o assistente virtual? \\
\hline
\end{tabular}

Quadro 1: Critérios de usabilidade e suas perguntas. Fonte: Os autores

\subsection{Perfil dos Participantes}

O grupo dos jovens adultos contou com 13 participantes do sexo masculino e sete do sexo feminino. O nível de escolaridade dos participantes, de acordo com o questionário, se configurou da seguinte maneira: nenhum participante possuía "ensino médio incompleto", apenas um com "ensino médio completo", nove com "ensino superior incompleto", oito com "ensino superior completo" e dois participantes com "pós-graduação incompleta".

O contato com a Internet, assim como a utilização de smartphones, foi unânime neste grupo, sendo que todos utilizam esses recursos, frequentemente, para realizar as mais diversas tarefas. Nesse grupo, o número de usuários que já havia utilizado um assistente virtual anteriormente foi de 12 pessoas. Destas, oito utilizam raramente um assistente virtual, três pessoas afirmaram usar às vezes e apenas um participante afirmou que sempre o utiliza para realizar tarefas no dia-a-dia.

O grupo dos participantes adultos se dividiu entre 10 homens e 10 mulheres. Com base no levantamento dos dados deste grupo, pode-se observar que um participante possuía "ensino médio completo", 13 tinham "ensino superior completo", um tinha "pós-graduação incompleta" e os outro cinco participantes tinham "pós-graduação completa". Assim como o grupo anterior, todos nesta faixa etária já tiveram contato com a Internet e smartphones. Todavia, houve uma variação da quantidade de uso do smartphone durante a semana, sendo que 12 afirmaram que sempre utilizam, sete são aqueles que utilizam muitas vezes, e uma pessoa que utiliza raramente. Nesse grupo, 13 participantes nunca tinham utilizado um assistente virtual antes e sete já utilizaram. Destes, seis disseram utilizar raramente esse tipo de ferramenta e um deles afirmou usar, às vezes, um assistente virtual para realizar algumas tarefas.

A divisão para o grupo dos idosos foi de oito participantes do sexo masculino e 12 do sexo feminino. Ao analisar os dados deste grupo etário, pode-se observar que dois participantes afirmaram ter "ensino médio incompleto", 10 participantes tinham "ensino médio completo", cinco tinham "ensino superior completo", e os outros três participantes tinham "pós-graduação completa". Em relação ao uso da Internet, 17 já tiveram algum tipo de contato com essa tecnologia em algum momento de suas vidas. Em relação ao uso de smartphones, este dispositivo é utilizado por quinze dos vinte participantes. Destes, dois utilizam raramente, nove participantes utilizam às vezes e os outros quatro utilizam sempre seus smartphones. Dos participantes desse grupo, 18 afirmaram que nunca utilizaram um assistente virtual para realizar qualquer tipo de tarefa e apenas dois alegaram já ter utilizado um assistente virtual. Dentre esses últimos, um utiliza a ferramenta de assistente virtual raramente e o outro utiliza ocasionalmente para auxiliar na realização de tarefas cotidianas.

\subsection{Ferramentas na Pesquisa de Campo}

Foram utilizados dois aparelhos smartphones, um para a realização das tarefas com a Siri (Iphone 5s com iOS 12.3.1) e outro para a execução do Google Assistant, sendo o modelo Galaxy A5 (2016) com Android 7.0. Os desempenhos dos participantes não foram influenciados pelo modelo do smartphone em uso. A versão utilizada de cada um dos assistentes virtuais foi a mesma para todos os participantes, e, também, a mais atual disponível para os dois sistemas operacionais. Durante a realização dos testes, foi utilizada a conexão $4 \mathrm{G}$ de cada um dos aparelhos, para que a rede wi-fi não 
interferisse nos desempenhos dos participantes. Ademais, foi criada uma conta fictícia no WhatsApp para que os participantes enviassem a mensagem solicitada na T5.

A disposição das tarefas realizadas nos assistentes virtuais ocorreu da seguinte maneira: a primeira (T1) solicitou que o participante adicionasse um alarme; na segunda tarefa (T2) o participante deveria consultar a previsão do tempo; a terceira tarefa (T3) solicitou que o participante procurasse por restaurantes na região; na quarta tarefa (T4) o participante deveria adicionar um lembrete pré-determinado; a última tarefa (T5) solicitou que o participante enviasse uma mensagem préestabelecida pelo Whatsapp. Todos os participantes receberam as orientações contendo os passos necessários para a execução das tarefas.

\section{DISCUSSÃO}

A análise dos dados foi realizada com base na comparação entre os três grupos definidos anteriormente e considerando os dois assistentes virtuais, em relação às cinco tarefas (T1, T2, T3, T4 e T5) executadas pelos participantes. A Figura 2 apresenta as médias dos tempos gastos por cada grupo ao realizar as tarefas nos dois assistentes virtuais.

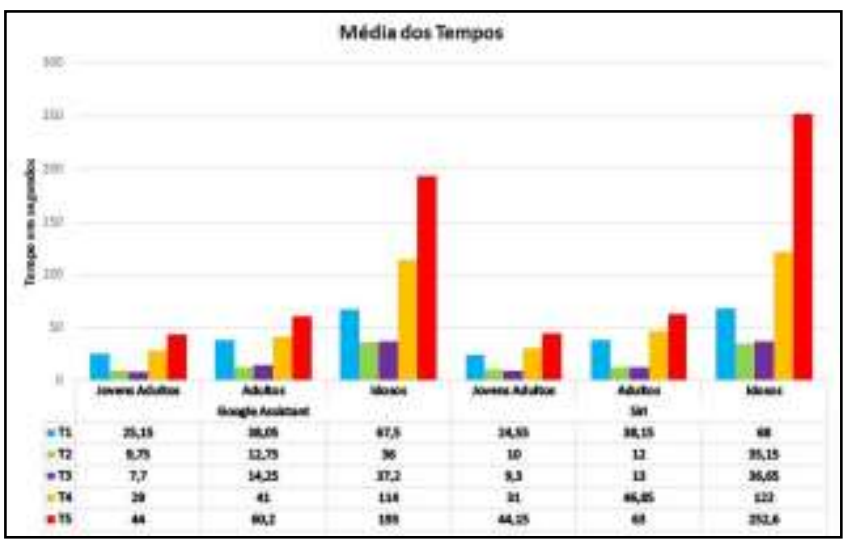

Figura 2: Comparação do tempo gasto entre os grupos em relação às tarefas propostas nos assistentes virtuais. Fonte: Os autores

O desempenho dos participantes idosos, em ambos assistentes, foi inferior se comparado às outras faixas etárias durante a primeira tarefa, levando aproximadamente o dobro do tempo dos adultos e o triplo do tempo dos jovens adultos. Já os adultos tiveram desempenho mediano dentre os grupos, tendo, em média, um tempo de 13 e 14 segundos (no Google Assistant e Siri, respectivamente) a mais do que os jovens adultos. Na T2 e na T3, os idosos diminuíram a diferença do tempo em relação aos adultos, com uma diferença média de 24 segundos para o Google Assistant e 23 segundos para a Siri. Analisando a T4, o desempenho dos idosos foi praticamente quatro vezes inferior quando comparado com o grupo dos jovens adultos, sendo que houve uma diferença média de oito segundos entre o Google Assistant e a Siri. Na T5, houve uma diferença de 59 segundos no grupo dos idosos entre os dois assistentes virtuais.

Vale ressaltar que, para a análise dos dados relacionados ao tempo gasto pelos participantes na execução das tarefas, foram excluídos da amostra alguns outliers, pois poderiam enviesar os resultados. Para isso, foi utilizado o método baseado na amplitude interquartil (IQR) para cada uma das tarefas, que consiste na diferença do terceiro quartil e do primeiro quartil. Com o IQR sendo uma estimativa de variabilidade, é possível calcular os limites inferior (Linf) e superior (Lsup). O limite inferior segue a fórmula $\operatorname{Linf}=$ média $-1,5$ * $I Q R$; já a fórmula utilizada para calcular o limite superior foi Lsup $=$ média $+1,5$ * $I Q R$, onde a média corresponde à média aritmética dos tempos de cada tarefa de cada grupo etário.

Em relação ao tempo de aprendizagem, os idosos julgaram que as duas últimas tarefas propostas foram mais difíceis (Figura 3). Ainda para essas duas tarefas, os idosos acharam que o assistente Siri não transmitiu as informações verbais de maneira compreensiva. Comparando os dois assistentes virtuais, os participantes julgaram que o Google Assistant apresenta as informações verbalmente de maneira mais fácil e clara. Já para as informações apresentadas na tela dos dispositivos, os dois assistentes obtiveram resultados similares.

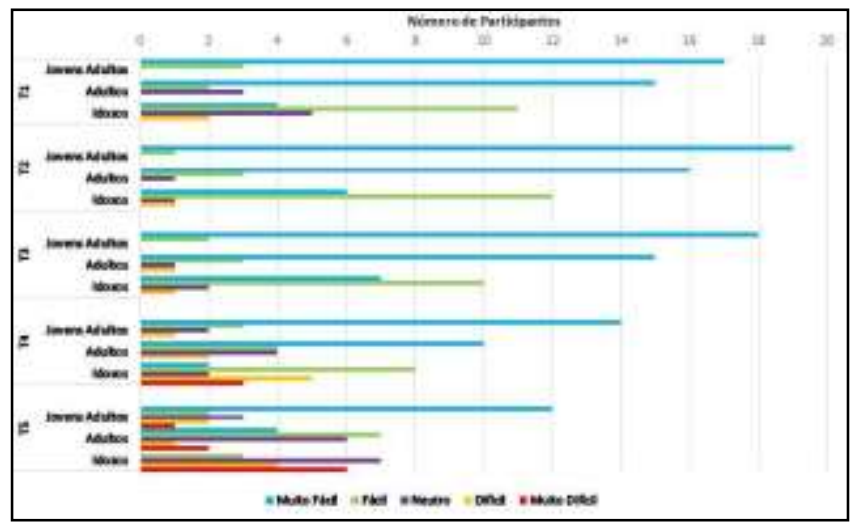

Figura 3: Facilidade de execução das tarefas propostas. Fonte: Os autores

No que tange ao desempenho, pode-se observar que os idosos realizaram as tarefas gastando um tempo maior do que os outros dois grupos, mas, apesar disso, consideraram que os assistentes virtuais agilizaram a realização das tarefas, já que esse grupo está ciente de suas limitações físicas e em relação à tecnologia, ou seja, poderiam gastar mais tempo para realizar as mesmas tarefas sem os assistentes virtuais. Já os outros grupos, por utilizarem mais os smartphones, tinham uma noção de quanto tempo gastariam para realizar as tarefas propostas e acharam, em algumas tarefas, que os assistentes não agilizaram sua execução. Deve-se destacar que, para os três grupos etários, todos os participantes que possuíam uma experiência prévia no uso de smartphones obtiveram melhor desempenho na realização 
das tarefas, bem como foram os que menos cometeram erros durante os testes de usabilidade. Muitos idosos afirmaram que um dos motivos de acharem que as tarefas foram realizadas de forma mais ágil, foi pelo fato de os assistentes interagirem de forma verbal com os usuários. Assim, os usuários não precisaram se preocupar em procurar por possíveis teclas e/ou botões, nem forçar a visão para visualizar a tela, mitigando várias limitações causadas pela velhice.

No que se refere ao terceiro critério avaliado (taxa de erros cometidos pelos usuários), dos oito idosos que já possuíam experiência prévia, três cometeram erro em uma tarefa. Nos três casos, esses erros ocorreram no sistema operacional que não estavam habituados. Para a taxa de erros cometidos ao utilizarem o Google Assistant, em todas as tarefas propostas, o grupo etário que mais cometeu algum tipo de erro foi o dos idosos. O grupo dos jovens adultos cometeu erros apenas na última tarefa, e, mesmo assim, a quantidade de jovens adultos que afirmou cometer um erro foi quatro vezes menor do que a quantidade de idosos. Já para a taxa de erros cometidos com o assistente Siri, o grupo de participantes idosos foi o que mais cometeu erros ao utilizar esse assistente, sendo que na primeira tarefa a quantidade de adultos e idosos foi igual; na terceira tarefa o número de adultos foi superior. Ao se observar o grupo dos jovens adultos, percebeu-se que eles cometeram erro em três tarefas. Uma possível razão para isto é a falta de familiaridade com dispositivos que utilizam iOS. Os dois assistentes obtiveram bom desempenho na recuperação de erros cometidos pelos usuários. Na opinião dos participantes, os assistentes permitiram refazer facilmente alguma ação cometida por engano.

$\mathrm{Na}$ análise da sedimentação do conhecimento por experiência, para o Google Assistant, apenas na T4 houve respostas discordando se o caminho percorrido pelo assistente era intuitivo, sendo de um adulto e dois idosos. Já para o assistente Siri, esse número foi um pouco maior, variando de um a quatro participantes para todas as tarefas, com exceção da T1. No geral, os participantes afirmaram conseguir realizar novamente as três primeiras tarefas com facilidade nos dois assistentes, entretanto, nas duas últimas tarefas, grande parte dos idosos e alguns adultos disseram não conseguir executar novamente.

No que diz respeito à satisfação subjetiva, comparando os dois assistentes em relação às suas interfaces, mais participantes acharam a interface da Siri mais agradável do que a do Google Assistant. Grande parte dos idosos disse preferir a interface da Siri, principalmente, por ser mais minimalista. Mesmo os idosos gastando mais tempo para realizar as tarefas do que os jovens adultos, a maioria afirmou que usaria novamente um assistente virtual. Para os jovens adultos, a maioria afirmou que voltaria a usar a Siri, principalmente pelo fato de a maioria desses participantes não estar acostumada com dispositivos iOS, logo, conseguiriam realizar as tarefas com mais facilidade. Para os adultos, a maioria afirmou que voltaria a usar o assistente virtual Google Assistant, mas por um motivo diferente ao dos jovens adultos. Neste caso, os adultos afirmaram que a chance de adquirirem um dispositivo da Apple é menor, por isso, mais adultos responderam que não utilizariam a Siri novamente.

\section{CONSIDERAÇÕES FINAIS}

Esse trabalho foi elaborado visando avaliar a inclusão e o desempenho de pessoas idosas no uso dos assistentes virtuais Google Assistant e Siri em comparação com pessoas mais jovens. Foi realizada uma análise da usabilidade desses assistentes, realizando cinco tarefas pré-determinadas, levando em conta as seguintes métricas: tempo de aprendizagem, desempenho, taxa de erros cometidos pelo usuário, sedimentação do conhecimento por experiência e satisfação subjetiva. Os perfis dos participantes voluntários da pesquisa foram bem diversificados, indo desde usuários que utilizavam com frequência o assistente virtual de seus smartphones até usuários que não costumavam utilizar smartphones no seu dia-a-dia. Os questionários foram aplicados após a interação dos participantes com os dois assistentes virtuais para cada grupo etário. Pode-se, então, analisar os resultados obtidos entre os grupos e entre os dois assistentes virtuais, assim como prever uma tendência dos comportamentos que se repetissem. O Quadro 2 sintetiza os principais resultados obtidos em cada um dos critérios analisados.

\begin{tabular}{|c|c|}
\hline Critério & Conclusões \\
\hline $\begin{array}{c}\text { Tempo de } \\
\text { Aprendizagem }\end{array}$ & $\begin{array}{l}\text { O grupo dos idosos foi o que teve mais dificuldade } \\
\text { em identificar e compreender as informações } \\
\text { apresentadas pelos assistentes virtuais. Estes } \\
\text { ressaltaram que devido ao fato de a interação com os } \\
\text { assistentes virtuais ser verbal, isso auxiliou na } \\
\text { realização das tarefas. }\end{array}$ \\
\hline Desempenho & $\begin{array}{l}\text { Como já esperado, os participantes idosos tiveram } \\
\text { desempenho inferior em relação aos outros } \\
\text { participantes. Os adultos, por sua vez, gastaram mais } \\
\text { tempo para concluir as mesmas tarefas realizadas } \\
\text { pelos jovens adultos. Outro fator que influenciou na } \\
\text { usabilidade foi a experiência prévia de uso da } \\
\text { tecnologia e a familiarização com smartphones. }\end{array}$ \\
\hline $\begin{array}{c}\text { Taxa de Erros } \\
\text { Cometidos } \\
\text { pelo Usuário }\end{array}$ & $\begin{array}{l}\text { A quantidade de erros cometidos pelos idosos foi } \\
\text { mais alta do que a dos outros grupos. Este critério } \\
\text { está fortemente atrelado às interações com o } \\
\text { assistente virtual para que o usuário realize a tarefa. } \\
\text { As tarefas consideradas mais difíceis foram as que } \\
\text { possuíam várias interações com o assistente virtual. } \\
\text { A perda de visão decorrente da idade fez com que a } \\
\text { compreensão das informações apresentadas na tela se } \\
\text { tornasse mais difícil, deste modo, os participantes que } \\
\text { conseguiam compreender as informações com mais } \\
\text { facilidade realizavam as tarefas mais rapidamente. }\end{array}$ \\
\hline $\begin{array}{l}\text { Sedimentação } \\
\text { do } \\
\text { Conhecimento } \\
\text { por } \\
\text { Experiência }\end{array}$ & $\begin{array}{l}\text { A maioria dos idosos achou o caminho percorrido } \\
\text { pelo assistente Google Assistant mais intuitivo do } \\
\text { que o da Siri. As respostas de todos os participantes } \\
\text { sobre não conseguirem realizar as tarefas novamente } \\
\text { se restringiram nas duas últimas tarefas (T4 e T5). }\end{array}$ \\
\hline $\begin{array}{c}\text { Satisfação } \\
\text { Subjetiva }\end{array}$ & $\begin{array}{l}\text { O grupo de idosos, mesmo cometendo mais erros, foi } \\
\text { o grupo que mais apresentou resultados positivos em } \\
\text { relação à utilização futura dos assistentes virtuais, } \\
\text { sendo que } 85 \% \text { dos idosos afirmou que utilizaria } \\
\text { novamente o Google Assistant e } 80 \% \text { a Siri. }\end{array}$ \\
\hline
\end{tabular}

Quadro 2: Principais resultados. Fonte: Os autores 


\section{AGRADECIMENTOS}

Os autores agradecem a todos os participantes que atuaram voluntariamente na realização desta pesquisa.

\section{REFERÊNCIAS}

[1] Organização das Nações Unidas - ONU (2014). https://news.un.org/en/story/2014/11/483012\#.VFyq6_nF-z4.

[2] Instituto Brasileiro de Geografia e Estatística - IBGE (2018) https://biblioteca.ibge.gov.br/visualizacao/livros/liv101631 informativo.pdf.

[3] Ministério da Saúde (2016). https://portalms.saude.gov.br/noticias/agenciasaude/25924-ministerio-recomenda-e-preciso-envelhecer-com-saude.

[4] Jakob Nielsen (2000). Designing web usability. Indianapolis: News Riders Publishing.

[5] Francisco Alexandre Ferreira Biscaia Godinho (2010). Uma nova abordagem para a formação de engenharia de reabilitação em Portugal. Tese de Doutorado. Universidade de Trás-os-Montes e Alto Douro. Engenharia Electrotécnica e de Computadores.

[6] Rodrigo de Rosso Krug, Eleonora d'Orsi, André Junqueira Xavier (2019). Associação entre o uso de internet e a função cognitiva de idosos, estudo longitudinal populacional Epifloripa Idoso. Revista Brasileira de Epidemiologia, São Paulo, 22.

[7] Chris M. Bleakley, Darryl Charles, Alison Porter-Armstrong, Michael D. J McNeill, Suzanne M. McDonough, Brendan McCormack (2015). Gaming for health: a systematic review of the physical and cognitive effects of interactive computer games in older adults. Journal of Applied Gerontology. 34(3).

[8] Nina Nevalainen, Katrine Riklund, Micael Andersson, Jan Erik Axelsson, Mattias Ögren, Martin Lövdén, Ulman Lindenberger, Lars Bäckman, Lars Nyberg (2015). Cobra: A prospective multimodal imaging study of dopamine, brain structure and function, and cognition. Brain Research. 1612, 83-103.

[9] Verena Klusmann, Andrea Evers, Ralf Schwarzer, Peter Schlattmann, Friedel M. Reischies, Isabella Heuser, Fernando C. Dimeo (2010). Complex mental and physical activity in older women and cognitive performance: a 6-month randomized controlled trial. Journal of Gerontology Series A Biological Sciences and Medical Sciences. 65(6), 680-688.

[10] Adele Diamond, Daphne S. Ling (2016). Conclusions about interventions, programs, and approaches for improving executive functions that appear justified and those that, despite much hype, do not. Developmental Cognitive Neuroscience, $18,34-48$.

[11] Irina Ivanova Stoyanova (2014). Ghrelin: a link between ageing, metabolism and neurodegenerative disorders. Neurobiology of Disease. 72-83.

[12] Cisco (2010). Older people, technology and community. https://www.cisco.com/c/dam/en_us/about/ac79/docs/wp/ps/Report.pdf.

[13] Adriana Holtz Betiol, Richard Faust, Walter Cybis (2010). Ergonomia e usabilidade. São Paulo: Novatec.

[14] Jakob Nielsen (2012). Usability 101: Introduction to Usability. https:/www.nngroup.com/articles/usability-101-introduction-to-usability/.

[15] Amanda Meincke Melo, Lara Schibelsky Godoy Piccolo, Ismael Mattos Andrade Ávila, Cláudia de Andrade Tambascia (2008). Usabilidade, acessibilidade e inteligibilidade aplicadas em interfaces para analfabetos, idosos e pessoas com deficiência. Proceedings of the VIII Brazilian Symposium on Human Factors in Computing Systems. Sociedade Brasileira de Computação, 354-355.

[16] Silvana Maria Affonso de Lara, Renata Pontin de Mattos Fortes, Cibele Maria Russo, Andre Pimenta Freire (2016). A study on the acceptance of website interaction aids by older adults. Universal Access in the Information Society, 15(3), 445-460.

[17] Jakob Nielsen, Hoa Loranger (2007). Usabilidade na Web: Projetando Websites com Qualidade. Rio de Janeiro: Elsevier. 406

[18] Jakob Nielsen (1993). Usability Engineering. Academic Press.

[19] Jakob Nielsen (1994).10 Usability Heuristics for User Interface Design. https://www.nngroup.com/articles/ten-usability-heuristics/

[20] Patrick W. Jordan (1998). An Introduction to Usability. Londres: Taylor \& Francis Ltda.

[21] ISO 9241-11 (1998). Ergonomics of human-system interaction - Part 11: Usability: Definitions and concepts.

[22] John Whiteside, John Bennett, Karen Holtzblatt (1988). Usability engineering: our experience and evolution. Handbook of Human-Computer Interaction, Helander M (ed). Elsevier.

[23] Nigel Bevan (1995). Usability is quality of use. Anzai \& Ogawa (eds) Proc. 6th International Conference on Human Computer Interaction, July. Elsevier. http://www.usability.serco.com/papers/usabis95.pdf.

[24] Jennifer Preece, Yvonne Rogers and Helen Sharp (2005). Design de interação: além da interação homem-computador. Porto Alegre: Bookman.
[25] Ben Schneiderman, Catherine Plaisant (2004). Designing the user interface: strategies for effective human-computer interaction. Addison Wesley Publishing Company.

[26] Enrico Bertini, Tiziana Catarci, Alan Dix, Silvia Gabrielli, Stephen Kimani, Giuseppe Santucci (2009). Appropriating heuristics evaluation for mobile computing. International Journal of Mobile Human Computer Interaction. $1(1), 20-41$.

[27] Arlene J. Astell, Maggie P. Ellis, Lauren Bernardi, Norman Alm, Richard Dye, Gary Gowans, Jim Campbell (2010). Using a touch screen computer to support relationships between people with dementia and caregivers. Interacting with Computers, 22, 267-275.

[28] Marija Mamolo, Sergei Scherbov (2009). Population Projections for Forty-Four European Countries: The Ongoing Population Ageing. European Demographic Research Papers.

[29] Niamh Caprani, Noel E. O'Connor, Cathal Gurrin (2012). Touch screens for the older user. IntechOpen.

[30] Sara J. Czaja, Neil Charness, Arthur D. Fisk, Christopher Hertzog, Sankaran N. Nair, Wendy A. Rogers, Joseph Sharit (2006). Factors predicting the use of technology: Finding from the Center for Research and Education on Aging and Technology Enhancement. Psychology and Aging, 21, 333-352.

[31] Neil Charness, Tiffany S. Jastrzembski (2009). Gerontechnology. Future interaction design II. P. Saariluoma \& H. Isomaki (eds.). 1-29

[32] Neil Charness, Walter R. Boot (2009). Aging and Information Technology Use: Potential and Barriers. Current Directions in Psychological Science. 18(5), 253 258.

[33] Sara J. Czaja, Neil Charness, Arthur D. Fisk, Wendy A. Rogers, Joseph Sharit (2009). Designing for older adults: Principles and creative human factors approaches.

[34] Don G. Bouwhuis (2003). Design for person-environment interaction in older age: a gerontechnological perspective. Gerontechnology. 2(3), 232-246.

[35] Michael Wooldridge, Nicholas R. Jennings (1995). Intelligent Agents: Theory \& Practice. Knowledge Engineering Review, 10(2), 115-152.

[36] Yasmine Arafa, Abe Mamdani (2000). Virtual personal service assistants: towards realtime characters with artificial hearts. ACM Intelligent User Interfaces Conference, New Orleans, USA.

[37] Eliseo Reategui, Alexandre Lorenzatti (2005). Um assistente virtual para resolução de dúvidas e recomendação de conteúdo. V Encontro Nacional de Inteligência Artificial, São Leopoldo.

[38] Robert S. Cooper, Jeff F. McElroy, Walter Rolandi, Derek Sanders, Richard M. Ulmer, Edward Peebles (2008). Personal virtual assistant. U.S. Patent n.6, $757,362 \mathrm{~B} 1$

[39] Matthew B. Hoy (2018). Alexa, Siri, Cortana, and more: an introduction to voice assistants. Medical Reference Services Quarterly. 37(1), 81-88.

[40] Google (2019). Google Assistant. https://assistant.google.com/

[41] Apple (2019). Siri. http://www.apple.com/ios/siri/

[42] Christian Stößel, HartmutWandke, Lucienne Blessing (2010). Gestural Interfaces for Elderly Users: Help or Hindrance?. Gesture in Embodied Communication and Human-Computer Interaction, 269-280.

[43] Sri Kurniawan (2008). Older people and mobile phones: A multi-method investigation. International Journal of Human-Computer Studies, 66(12), 889901.

[44] Atsuo Murata, Hirokasu Iwase (2005). Usability of touch-panel interfaces for older adults. Human Factors The Journal of the Human Factors and Ergonomics Society, 47(4), 767-776.

[45] Gustavo López, Luis Quesada, Luis A. Guerrero (2017). Alexa vs. Siri vs Cortana vs. Google Assistant: A comparison of speech-based natural user interfaces. Advances in Human Factors and Systems Interaction, 241-250.

[46] Thiago Silva Chiaradia, Rodrigo Duarte Seabra, Adriana Prest Mattedi (2018) Avaliando a usabilidade do assistente virtual Siri em dispositivos móveis com ênfase em usuários idosos. Anais do Computer on the Beach, 572-781.

[47] Beatriz Hencklein Giassi, Rodrigo Duarte Seabra (2018). Usability assessment of the Instagram application on smartphones with emphasis on elderly users. XVIII Brazilian Symposium on Human Factors in Computing Systems.

[48] Elizabeth A. Bosman, Neil Charness (1996). Age differences in skilled performance and skill acquisition. Perspectives on Cognitive Chance in Adulthood and Aging, 428-453. 